\title{
Efecto del aprovechamiento forestal sobre la estructura y diversidad arbórea en selvas tropicales de Quintana Roo, México
}

\author{
Effect of logging on tree structure and diversity in tropical forests of Quintana Roo, Mexico
}

\author{
Alfredo Esteban Tadeo-Noble a*, Juan Ignacio Valdez-Hernández a, \\ Leonardo Beltrán-Rodríguez a ${ }^{\text {, Edmundo García-Moya a }}$ \\ *Autor de correspondencia: a Colegio de Postgraduados, Campus Montecillo, Carretera México-Texcoco km 36,5 Montecillo, \\ Estado de México, México, C.P. 56230, tel.: 52-595-95-20200, alfredotadeo@gmail.com
}

\begin{abstract}
SUMMARY
Timber harvesting may lead to changes in the dasonomic and ecological attributes of tree vegetation, affecting the productivity and biodiversity of ecosystems. Although, little is known about the effect of this practice on the tropical forests of Mexico. The aim was to compare the structure and diversity in forest ejidos under timber harvesting in Quintana Roo. Two inventories (2009) were developed in Permanent Forest Areas (PFA) of medium evergreen forests. In Bacalar, 625 plots of 10x25 m distributed in 15.6 ha were used, while in Noh Bec 302 plots of 10x40m located in 48.32 ha. In each plot, all trees with normal diameter $\geq 7.5 \mathrm{~cm}$ were measured to document richness and dasometric variables. Structural indexes (IVI and FMVI) and diversity indexes (Shannon-Wiener $H^{\prime}$ and Sorensen CS) were also calculated. In Bacalar there were 86 tree species (36 families and 81 genera), 284 tree ha $^{-1}, 7.3 \mathrm{~m}^{2} \mathrm{ha}^{-1}$ of BA and $86.4 \mathrm{~m}^{3} \mathrm{ha}^{-1}$ of TTV; whereas in Noh Bec, we registered 106 species ( 34 families and 94 genera), 625 individuals ha ${ }^{-1}, 23.9 \mathrm{~m}^{2} \mathrm{ha}^{-1}$ of BA and $183.8 \mathrm{~m}^{3} \mathrm{ha}^{-1}$ of TTV. Bursera simaruba (IVI= $16.6 \%$, FMVI $=18.1 \%$ ) was the dominant species in Bacalar, whereas in Noh Bec it was Pouteria reticulata (IVI= $13.5 \%, \mathrm{FMVI}=14.8 \%)$. The species diversity was not different $(P>0.05)$ between ejidos $\left(H^{\prime}=3.48\right.$ vs $\left.H^{\prime}=3.37\right)$; nonetheless there was a high floristic similarity $(C s=60.4 \%)$. Our results provide evidence that timber harvesting associated with the history of natural disturbances drives a key role in the structure and diversity of tropical forests.
\end{abstract}

Key words: Bacalar, Shannon-Wiener and Sorensen indexes, Noh Bec, Importance value index, Forest management value index.

\section{RESUMEN}

El aprovechamiento maderable puede ocasionar modificaciones en atributos ecológicos y dasonómicos de la vegetación arbórea, afectando la productividad y biodiversidad de los ecosistemas; sin embargo, poco se conoce del efecto en selvas tropicales de México. El objetivo de este estudio fue comparar la estructura y diversidad en selvas ejidales bajo aprovechamiento forestal en Quintana Roo. Fueron desarrollados dos inventarios (2009) en áreas forestales permanentes de selva mediana subperennifolia: en Bacalar se aplicaron 625 unidades de muestreo (10x25 m distribuidas en 15,6 ha) y en Noh Bec 302 unidades (10x40 m ubicadas en 48,32 ha). En ellas se midieron todos los árboles con diámetro normal $\geq 7,5 \mathrm{~cm}$ para documentar la riqueza y variables dasométricas. Se estimaron índices estructurales (IVI e IVMF) y de diversidad (Shannon-Wiener H' y Sorensen Cs). En Bacalar se registraron 86 especies arbóreas (36 familias, 81 géneros), 284 individuos $\mathrm{ha}^{-1}, 7,3 \mathrm{~m}^{2} \mathrm{ha}^{-1}$ de $\mathrm{AB}$ y volumen de $86,4 \mathrm{~m}^{3} \mathrm{ha}^{-1}$; en Noh Bec 106 especies (34 familias, 94 géneros), 625 individuos ha ${ }^{-1}, 23,9 \mathrm{~m}^{2} \mathrm{ha}^{-1} \mathrm{de} \mathrm{AB}$ y volumen de $183,8 \mathrm{~m}^{3} \mathrm{ha}^{-1}$. Bursera simaruba $(\mathrm{IVI}=16,6 \% ; \mathrm{IVMF}=18,1 \%)$ fue la especie dominante en Bacalar, y en Noh Bec fue Pouteria reticulata (IVI $=13,5 \%$; IVMF $=14,8 \%$ ). La diversidad de especies no fue diferente entre ejidos; no obstante, la semejanza florística fue alta (Cs $=60,4 \%)$. Estos resultados proveen evidencia de que el aprovechamiento maderable asociado al historial de disturbios naturales tiene un papel determinante sobre la estructura y diversidad de las selvas secundarias estudiadas.

Palabras clave: Bacalar, Shannon-Wiener, Sorensen, Noh Bec, valor de importancia.

\section{INTRODUCCIÓN}

La pérdida y fragmentación de las selvas es considerada una de las principales amenazas para su estabilidad y permanencia, debido a que estos ecosistemas son reconocidos a nivel mundial por albergar una elevada riqueza de grupos funcionales y especies (Chazdon 2013). Los principales factores de transformación de las selvas son el cambio de uso de las tierras para la agricultura y la ganadería, el crecimiento poblacional, la infraestructura de carreteras y el aprovechamiento maderable de tipo selectivo, en particular de especies arbóreas con maderas duras-preciosas (Bray 2004). También se ha sugerido que la pérdida biológica y de servicios ambientales en estos ecosistemas 
obedece, entre otros causales, a fenómenos naturales como huracanes (Whigham et al. 2003). En ambos casos, disturbio humano o natural, existe una alteración de la composición original de especies arbóreas, derivada de la respuesta ecológica adaptativa ante ciertos disturbios por grupos funcionales (Chazdon 2013).

La estructura de una selva representa el arreglo espacial (horizontal y vertical) y el ordenamiento temporal de los elementos que lo constituyen (Gadow et al. 2007). La diversidad, en cambio, es una medida que expresa la riqueza de especies y la repartición de los individuos entre estas (Magurran y McGill 2014). Gadow et al. (2007) señalan que la estructura de las selvas se caracteriza tanto por el número de especies como por la distribución de las dimensiones de los árboles. Así, a mayor heterogeneidad de la estructura espacial incrementa la riqueza de especies y se alcanza la estabilidad ecológica (Günter et al. 2011). Pero, en bosques bajo aprovechamiento maderable, las operaciones silvícolas modifican la estructura forestal y, por lo tanto, tienen un potencial importante en asegurar la diversidad y funciones ecológicas de las masas (Günter et al. 2011).

La caracterización de la estructura forestal generalmente se basa en índices cuantitativos aplicados a ecosistemas puros o modificados (Gadow et al. 2007), los cuales históricamente se han estimado mediante la adición de parámetros exclusivamente del plano horizontal (abundancia, frecuencia y dominancia) (Magurran y McGill 2014); y recientemente incluyen variables tanto horizontales como verticales (diámetro, altura y cobertura) (Carreón y Valdez 2014). No obstante, estos índices solo aportan información sobre la dominancia estructural de las especies con fines de conservación, pero no incluyen criterios de interés para selvas bajo aprovechamiento maderable (p. ej. volumen cosechable).

El manejo forestal en selvas consiste en la aplicación de principios técnicos en las operaciones de producción, bajo una perspectiva de aprovechamiento sostenido de las especies de interés comercial (Gadow et al. 2007, Günter et al. 2011). Los efectos del manejo forestal sobre la estructura de las selvas tropicales han sido estudiados por diversos autores en diferentes partes del planeta (Villela et al. 2006, Adekunle et al. 2010, Imai et al. 2012). Algunas de estas investigaciones demuestran que el aprovechamiento maderable afecta la repoblación natural, atributos estructurales, riqueza y composición de especies; particularmente por el derribo y arrastre del arbolado, que incide en la generación de claros y detona cambios en la distribución espacial y la dinámica de las comunidades (Finegan y Camacho 1999, Guariguata y Ostertag 2001).

Particularmente en México, los esfuerzos de investigación sobre la sinecología de las selvas tropicales se han concentrado en el sureste del país (Koleff et al. 2012). Las selvas medianas y altas subcaducifolias y subperennifolias de la Península de Yucatán han sido de las mejor estudiadas en cuanto a riqueza y composición de especies en esta región (Zamora et al. 2008, Gutiérrez-Báez et al. 2011, Dzib et al. 2014); mientras que las selvas altas perennifolias han recibido menor atención académica (Bongers et al. 1988, Ibarra et al. 1995). El estado de Quintana Roo, ubicado en dicha Península, aun cuando posee el mayor número de reservas con selvas tropicales en México y tiene una larga historia de manejo forestal (Bray 2004), cuenta con un limitado número de investigaciones sobre la estructura de selvas sin aprovechamiento maderable (Navarro et al. 2012, Carreón y Valdez 2014), y menos aún sobre selvas intervenidas (Macario et al. 1995, Gutiérrez-Granados et al. 2011). La ausencia de esta información limita prácticas silvícolas sostenibles en los bosques tropicales con fines de manejo y conservación.

Los objetivos de este trabajo son: i) determinar y comparar la estructura y diversidad de especies arbóreas entre dos selvas ejidales de Quintana Roo sujetas a aprovechamiento forestal maderable, y ii) desarrollar un nuevo índice estructural para estimar la importancia silvícola de especies arbóreas con potencial de aprovechamiento maderable. Se espera que exista un efecto del aprovechamiento forestal sobre la estructura y diversidad en ambas comunidades, y que, debido a la semejanza ambiental y a un historial de intervención forestal semejante entre sitios de estudio, los parámetros estructurales y los estimadores de diversidad sean iguales.

\section{MÉTODOS}

Área de estudio. El estudio se llevó a cabo en los ejidos de Bacalar y Noh Bec, en el estado de Quintana Roo, México, en los cuales se desarrollan actividades de aprovechamiento forestal maderable. El ejido Bacalar cuenta con 51.919,4 ha de extensión territorial y se ubica en el municipio de Bacalar, en tanto que el ejido Noh Bec posee 24.122 ha y se ubica en el municipio de Felipe Carrillo Puerto; la distancia entre estas dos localidades es de $\sim 70 \mathrm{~km}$ (figura 1). En ambos ejidos el relieve es plano, con inclinación no mayor a $1 \%$ de pendiente, y altitud media de $10 \mathrm{~m}$ s.n.m. (INEGI 2017).

El clima de Bacalar es cálido subhúmedo (Aw1) con lluvias en verano, mientras que en Noh Bec las lluvias se prolongan hasta el invierno (Aw1 ( $\left.\mathrm{x}^{\prime}\right)$ ). El intervalo de temperatura en ambos ejidos varía entre 18 y $26^{\circ} \mathrm{C}$, y la precipitación anual promedio es de $1.200 \mathrm{~mm}$ (INEGI 2017). La vegetación en el área forestal permanente de Bacalar corresponde a 4.036,55 ha de selva mediana subperennifolia primaria y secundaria, con dominancia de una composición florística y una cubierta forestal características de sitios perturbados. El área forestal permanente de Noh Bec dispone de 18.000 ha de este mismo tipo de vegetación (Pennington y Sarukhán 2005), pero con elementos arbóreos de mayor porte. Los suelos dominantes en Bacalar y Noh Bec son: vertisoles, leptosoles y gleysoles (INEGI 2017).

Los aprovechamientos forestales en los ejidos Bacalar y Noh Bec estuvieron concesionados a empresas privadas de 1953 a 1983, fecha en la que surgió el Plan Piloto Forestal que motivó el aprovechamiento forestal comunitario 
(Bray 2004); razón por lo cual Bacalar y Noh Bec tienen 65 años de aprovechamiento forestal maderable comercial con programas de manejo forestal. El ejido Bacalar tiene autorizado un volumen de 10,8 $\mathrm{m}^{3} \mathrm{ha}^{-1}$ (entre 2010-2019) y el ejido Noh Bec de 23,9 $\mathrm{m}^{3}$ ha $^{-1}$ (2011-2015). En ambos ejidos se ha utilizado el método de manejo de selección bajo un sistema con tres ciclos de cosecha (policíclico), con caoba (Swietenia macrophylla King) como especie guía, bajo diámetros normales mínimos de corta $\geq 55 \mathrm{~cm}$ (Bray 2004).

Procedimiento de muestreo. En el ejido Bacalar durante el 2009 se establecieron con arranque aleatorio y de manera sistemática 625 unidades de muestreo (UM) de $250 \mathrm{~m}^{2}$ $(10 \times 25 \mathrm{~m})$ dispuestas en 15,6 ha a manera de cuadrantes, como lo sugiere Velasco et al. (2003), y se midieron todos los individuos con diámetros normales $(\mathrm{DN}) \geq \mathrm{a} 7,5 \mathrm{~cm}$ registrados en cada UM (figura 2A). En el mismo año, en el ejido Noh Bec se utilizó un diseño de muestreo por conglomerados distribuidos de manera sistemática en 48,32 ha., similar al utilizado en el Inventario Nacional Forestal y de Suelos de México (CONAFOR, 2012). Los conglomerados comprendieron 302 unidades de muestreo primario (UMP) de $1.600 \mathrm{~m}^{2}$, que incluyeron cuatro unidades de muestreo secundario (UMS1) de $200 \mathrm{~m}^{2}(10 \times 20 \mathrm{~m})$ donde se midieron los individuos con $\mathrm{DN} \geq 7,5 \mathrm{~cm}$, y otras cuatro unidades de muestreo secundario (UMS2) de $200 \mathrm{~m}^{2}$ donde se midieron los árboles con $\mathrm{DN} \geq 24,9 \mathrm{~cm}$ (figura 2B). El diseño de muestreo difirió entre ejidos de-

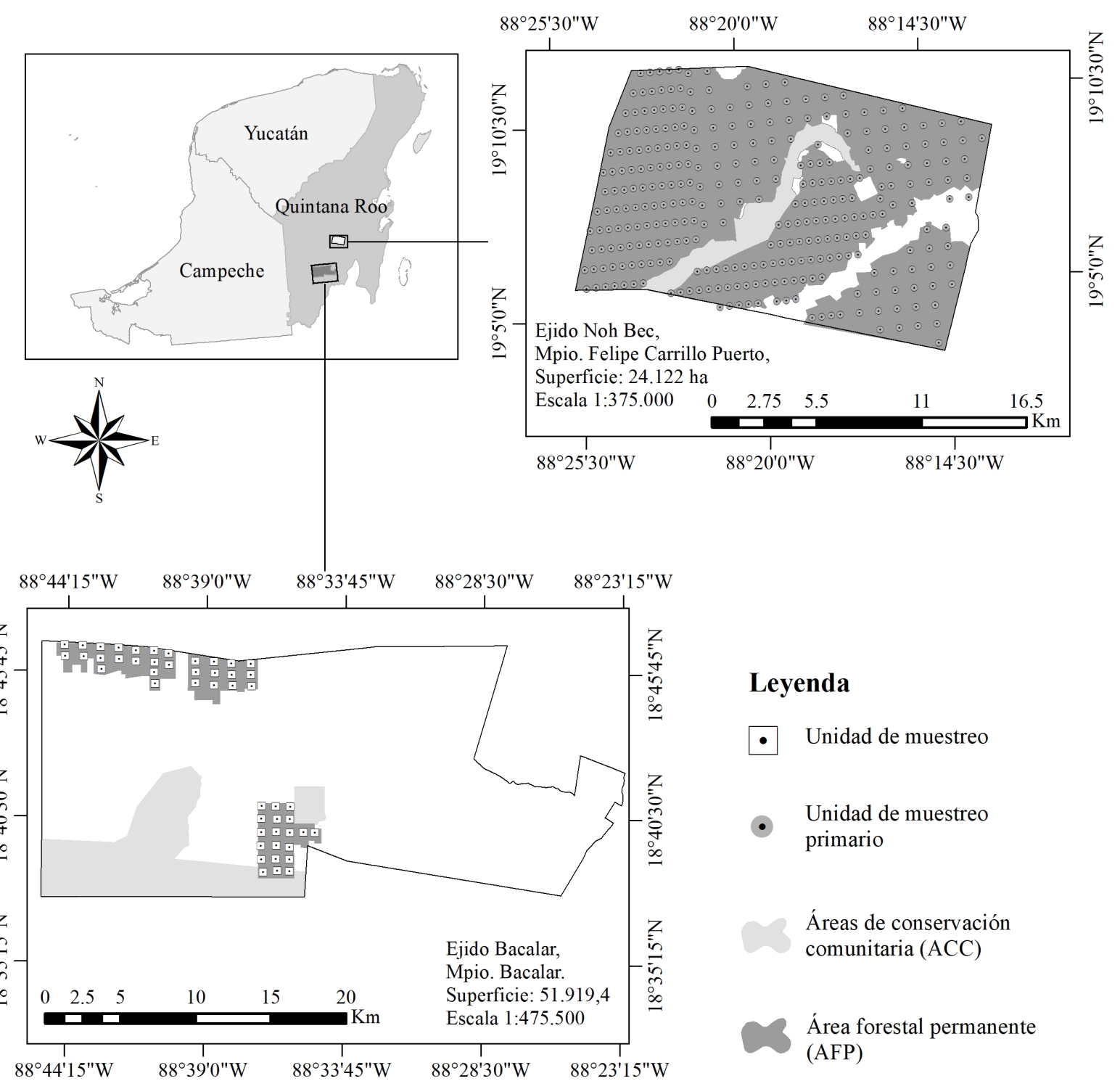

Figura 1. Ubicación geográfica de los ejidos Bacalar y Noh Bec, Quintana Roo, México.

Geographical location of the Bacalar and Noh Bec ejidos, Quintana Roo, Mexico. 
bido a requerimientos diferentes para la elaboración de cada programa de aprovechamiento forestal maderable. No obstante, la intensidad de muestreo fue semejante, con $0,4 \%$ de la superficie total $(4.036,55 \mathrm{ha})$ de su área forestal permanente en Bacalar y 0,3\% en Noh Bec (18.000 ha).

Colecta y determinación del material vegetal. Se recolectaron por triplicado ejemplares vegetales de cada especie para su determinación taxonómica por los especialistas en el Herbario "CHAPA" del Colegio de Postgraduados Campus Montecillo, México. La nomenclatura se estandarizó con base en Tropicos.org del Missouri Botanical Garden.

Estratificación vertical y horizontal. La estratificación vertical y categorías diamétricas se determinaron con base en la inflexión de una curva generada entre el número de individuos contra la altura y el diámetro, lo que permitió establecer el número de agrupaciones posibles. La agrupación estadística más apropiada $(P \leq 0,0001)$ para los datos se evaluó con un análisis de conglomerados con el método de partición basada en medioides. Para el caso se seleccionó el mayor coeficiente de silueta, que mide el grado de vínculo dentro de cada agrupamiento con la mejor distribución de los objetos agrupados (nj) y los coeficientes de agrupamiento interno (cj) dentro de los grupos (Kaufman y Rousseeuw 2005).

Importancia estructura. El valor de importancia estructural de las especies del componente arbóreo se determinó mediante el índice de valor de importancia expresado en porcentaje (ecuación 1), que contribuye a comprender cuáles de las especies presentes en la comunidad son estructu- ralmente dominantes mediante el cálculo de tres atributos ecológicos (densidad, dominancia y frecuencia) (Magurran y McGill 2014):

$$
\mathrm{IVI}=\left(\mathrm{Dr}+\mathrm{D}_{\mathrm{o}} \mathrm{r}+\mathrm{Fr}\right) / 3
$$

Donde, $\mathrm{IVI}=$ Índice de valor de importancia. $\mathrm{Dr}=$ Densidad relativa. $\mathrm{D}_{\mathrm{o}} \mathrm{r}=$ Dominancia relativa. $\mathrm{Fr}=$ Frecuencia relativa.

También se presenta por primera vez el índice de valor para el manejo forestal (IVMF), el cual es una propuesta teórica y metodológica del presente estudio generada a partir de la modificación del IVI. El IVMF estima la importancia silvícola de las especies arbóreas con condiciones deseables de aprovechamiento forestal en selvas. Este índice utilizado con el método de regulación puede simular los cambios estructurales futuros, en función del crecimiento y la dinámica de la selva. En el caso del IVI, la frecuencia relativa es reiterativamente obsoleta debido a que las especies más importantes son muy frecuentes (Magurran y McGill 2014). Por el contrario, la adición del volumen total del árbol (VTA) como variable combinada en el IVMF supone que todas las especies tienen el mismo volumen, como el índice de homogeneidad (Magurran y McGill 2014).

A diferencia de otros índices, el IVMF busca evaluar en dos planos dimensionales a una comunidad forestal. En un primer plano, el horizontal, la densidad (promedio por hectárea relativo) definida como el número de individuos por unidad de superficie y el área basal estimada como el diámetro a la altura del pecho. En un segundo plano, el vertical, el volumen total árbol es la expresión de la rela-
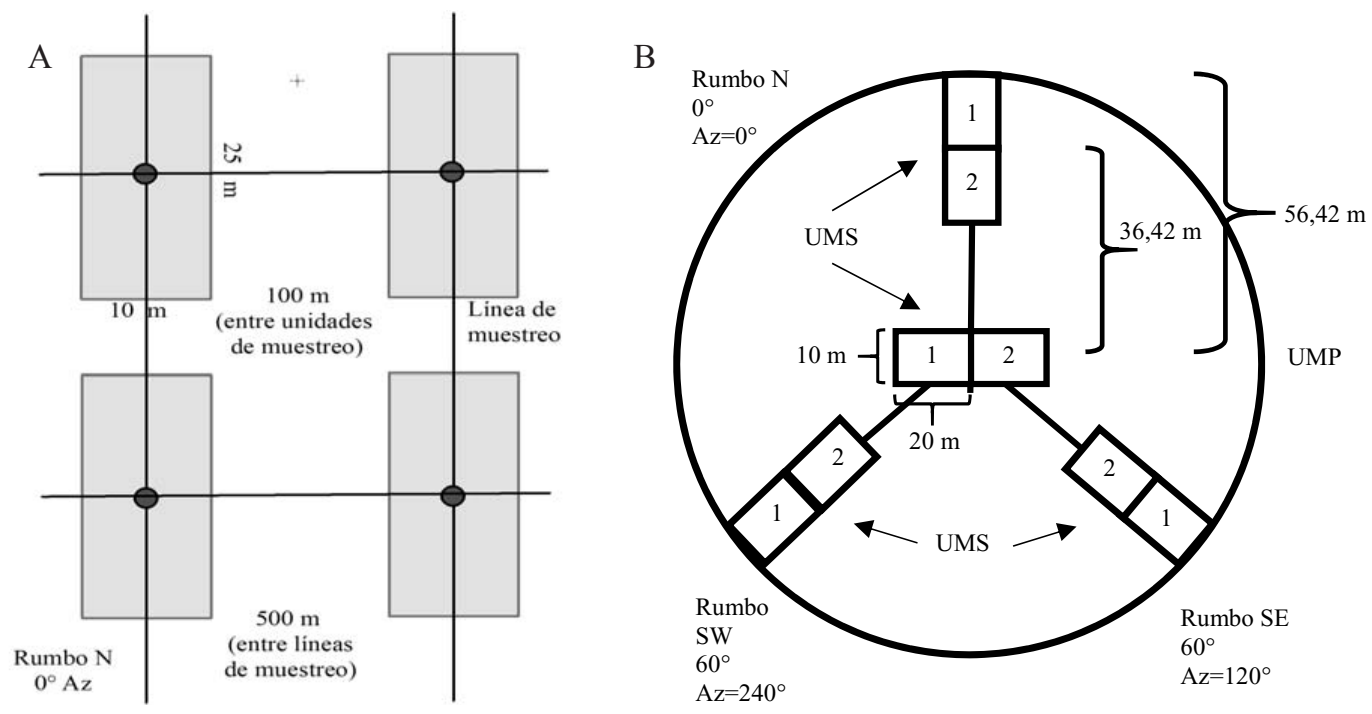

Figura 2. Esquema de muestreo utilizado en el inventario forestal de los ejidos Bacalar (A) y Noh Bec (B). UMP: unidad de muestreo primario; UMS1: unidad de muestreo secundario para árboles con $\mathrm{DN} \geq 7,5 \mathrm{~cm}$; UMS2: unidad de muestreo secundario para árboles con $\mathrm{DN} \geq 24,9 \mathrm{~cm}$.

Sampling scheme used in the forest inventory of the Bacalar (A) and Noh Bec (B) ejidos. UMP: primary sampling unit; UMS1: secondary sampling unit for trees with $\mathrm{DN} \geq 7.5 \mathrm{~cm}$; UMS2: secondary sampling unit for trees with $\mathrm{DN} \geq 24.9 \mathrm{~cm}$. 
ción entre altura del árbol por el área que ocupa. El IVMF se expresa en porcentaje y estima con la ecuación 2:

$$
\mathrm{IVMF}=\left(\mathrm{Dr}+\mathrm{D}_{\mathrm{o}} \mathrm{r}+\mathrm{Vr}\right) / 3
$$

Donde, $\mathrm{IVMF}=$ Índice de valor de manejo forestal. $\mathrm{Dr}=$ Densidad relativa. $\mathrm{D}_{\mathrm{o}} \mathrm{r}=$ Dominancia relativa. $\mathrm{Vr}=$ Volumen total árbol relativo.

Riqueza de especies. El índice de Margalef (Magurran y McGill 2014) se calculó con la ecuación 3 y calcula la riqueza de especies:

$$
\mathrm{D}_{\alpha}=(\mathrm{S}-1) / \ln (\mathrm{N})
$$

Donde, $D_{\alpha}=$ Índice de Margalef. $\mathrm{S}=$ Número de especies. $\ln (\mathrm{x})=\operatorname{logaritmo}$ natural. $\mathrm{N}=$ Número total de individuos. A mayor $\mathrm{D} \alpha$ mayor riqueza de especies.

Diversidad de especies. La heterogeneidad se calculó mediante el índice de Shannon-Wiener, que mide la uniformidad de la distribución de los individuos entre las especies en la muestra, por lo que toma en cuenta riqueza y abundancia (ecuación 4) (Magurran y McGill 2014):

$$
\mathrm{H}^{\prime}=-\sum(\mathrm{pi} \times \ln (\mathrm{pi}))
$$

Donde, H' = Índice de Shannon-Wiener. pi = Proporción de individuos del total de la muestra que corresponde a la i-ésima especie, es decir, el número de individuos de una especie dividido entre el número de individuos de todas las especies. $\ln (\mathrm{x})=$ Logaritmo natural.

Se utilizó la "t modificada" de Hutcheson (ecuación 5), con el propósito de comprobar la existencia de diferencias en la diversidad entre zonas de estudio (Magurran y McGill 2014).

$$
\mathrm{t}=\left(\mathrm{H}_{1}-\mathrm{H}_{2}\right) /\left(\operatorname{VarH}_{1}+\operatorname{VarH}_{2}\right)^{1 / 2}
$$

La varianza de las H' en cada predio se determinó mediante la ecuación 6 :

$\operatorname{Var} \mathrm{H}^{\prime}=\left[\left(\sum\left(\mathrm{pi} \times(\ln (\mathrm{pi}))^{2}\right)-\left(\sum(\mathrm{pi} \times \ln (\mathrm{pi}))\right)^{2}\right] / \mathrm{N}\right)+\left((\mathrm{S}-1) / 2 \times \mathrm{N}^{2}\right)[6]$

Con el valor t se calcularon los grados de libertad (ecuación 7) y se realizó la prueba de hipótesis.

$$
\mathrm{df}=\left(\operatorname{VarH}_{1}+\operatorname{VarH}_{2}\right)^{2} /\left(\left(\operatorname{VarH}_{1} / \mathrm{N}_{1}\right)^{2}+\left(\operatorname{VarH}_{2} / \mathrm{N}_{2}\right)^{2}\right)
$$

Donde, $\mathrm{H}_{\mathrm{i}}=$ Índice de Shannon-Wiener de las comunidades. Var H' = Varianzas de Shannon-Wiener de las comunidades. $\mathrm{N}_{\mathrm{i}}=$ Número total de individuos en cada comunidad.

El índice de diversidad alfa de Fisher (Magurran y McGill 2014), se estimó utilizando la ecuación 8. La estima- ción de la varianza alfa de Fisher se hizo con la ecuación 9 y dado que los valores de $\alpha$ de Fisher se distribuyen de manera normal, es posible estimar los intervalos de confianza al 95 \% (ecuación 10) (Magurran y McGill 2014).

$$
\mathrm{S}=\alpha \times \ln (1+(\mathrm{N} / \alpha)
$$

$$
\left.\mathrm{V}(\alpha)=\alpha^{3}\left[(\mathrm{~N}+\alpha)^{2} \times \ln ((2 \mathrm{~N}+\alpha) /(\mathrm{N}+\alpha))-(\alpha \mathrm{N})\right] /(\mathrm{SN}+\mathrm{S} \alpha-\mathrm{N} \alpha)^{2}\right)[9]
$$

$$
\mathrm{IC}=\alpha \pm 1,96 \times \sqrt{ }(\mathrm{V}(\alpha)
$$

Donde, $\alpha=$ Índice de alfa de Fisher. $\mathrm{V}(\alpha)=$ Varianza del alfa de Fisher. $\mathrm{N}=$ el número de individuos en la muestra. $\ln (\mathrm{x})=$ Logaritmo natural.

De manera que, si los valores de $\alpha$ de Fisher entre el límite inferior y superior del ejido Bacalar se traslapan con los intervalos de alfa de Fisher del ejido Noh Bec, se asume que la diversidad es semejante.

Repartición de especies. La repartición de las especies se estimó con la ecuación 11, y cuando expresa valores próximos a cero indican la dominancia de una especie, y valores cercanos a uno describen a especies en proporciones similares (Magurran y McGill 2014).

$$
\mathrm{E}=\mathrm{H}^{\prime} / \ln (\mathrm{S})
$$

Donde, $\mathrm{E}=$ =Índice de repartición de especies. H'= Índice de Shannon-Wiener. $\ln (\mathrm{x})=$ Logaritmo natural.

Semejanza floristica entre comunidades. El índice de Sorensen (Cs) entre las dos localidades se estimó con la ecuación 12 y utilizando datos de presencia y ausencia de las especies (Magurran y McGill 2014).

$$
\mathrm{C}_{\mathrm{s}}=(2 \mathrm{C} /(\mathrm{A}+\mathrm{B})) \times 100
$$

Donde, $\mathrm{C}_{\mathrm{s}}=$ Índice de Sorensen, en porcentaje. $\mathrm{A}=$ Número total de especies presentes en la comunidad A. $\mathrm{B}=$ Número total de especies presentes en la comunidad B. $\mathrm{C}=$ Número de especies comunes en ambas comunidades.

\section{RESULTADOS}

Riqueza, composición y atributos estructurales. En el área forestal permanente del ejido Bacalar se registraron 86 especies arbóreas, distribuidas en 36 familias y 81 géneros. Cinco familias concentran el $46,9 \%$ del total de la riqueza de especies en la comunidad: Fabaceae (17 especies), Rubiaceae (8 especies), Sapotaceae (6 especies), Malvaceae (4 especies) y Sapindaceae (4 especies); mientras que las 31 familias restantes acumulan el 53,1\%. Los géneros mejor representados en cuanto a número de especies fueron Lonchocarpus (3,5\%), Pouteria (2,3 \%), Byrsonima (2,3\%) y Psidium (2,3\%). En total se censaron 4.541 árboles 
(284 individuos ha-1), que aportan 7,3 $\mathrm{m}^{2} \mathrm{ha}^{-1}$ de $\mathrm{AB} y$ $86,4 \mathrm{~m}^{3} \mathrm{ha}^{-1}$ de volumen total.

En el área forestal permanente del ejido Noh-Bec se encontraron 106 especies arbóreas pertenecientes a 34 familias y 94 géneros. Las familias con mayor riqueza de especies fueron: Fabaceae (20,2 \%), Malvaceae (8,5\%), Sapindaceae $(7,4 \%)$, Rubiaceae $(6,4 \%)$ y Sapotaceae (5,3\%); en tanto que 29 familias restantes concentran el 52,1\% de la riqueza total registrada. Los géneros dominantes por riqueza de especies fueron Coccoloba (3,8 \%), Lonchocarpus (3,5 \%), Eugenia (2,8\%) y Caesalpinia (1,9\%). Se registraron en total 19.101 árboles en este ejido (625 individuos ha-1), con un $\mathrm{AB}$ de $23,9 \mathrm{~m}^{2} \mathrm{ha}^{-1}$ y $183,8 \mathrm{~m}^{3} \mathrm{ha}^{-1}$ de volumen total.

Estratificación vertical. El área forestal permanente Bacalar tuvo cuatro estratos: superior, dos intermedios e inferior (coeficiente de silueta de 0,54) (figura 3A). La altura promedio de los árboles fue de $12,3 \mathrm{~m}$, con algunos indi- viduos mayores a $15 \mathrm{~m}$. Los primeros estratos estuvieron constituidos por categorías de altura menores a 13,45 m, que representan alrededor del $90 \%$ del total de individuos por hectárea. La altura promedio de los árboles en el área forestal permanente Noh Bec fue de 12,0 m, con una baja proporción de árboles mayores a $20 \mathrm{~m}$. Se diferenciaron tres estratos: superior, intermedio e inferior (coeficiente de silueta de 0,56) (figura 3B). El estrato inferior se conformó por categorías de altura menores a $12,35 \mathrm{~m}$, que representa $61,6 \%$ del total de individuos por hectárea.

Distribución diamétrica. Los valores en DN en el área forestal permanente Bacalar variaron de $7,5 \mathrm{~cm}$ hasta $85 \mathrm{~cm}$, con una media de $21,7 \mathrm{~cm}$ y 284 individuos por hectárea. Se identificaron cuatro categorías diamétricas: dos delgadas $(7,45$ - 37,05) y dos gruesas $(37,06$ - 80,05) (coeficiente de silueta de 0,55 ) (figura 4A). La categoría delgada concentra casi el $85 \%$ del número de árboles por hectárea.
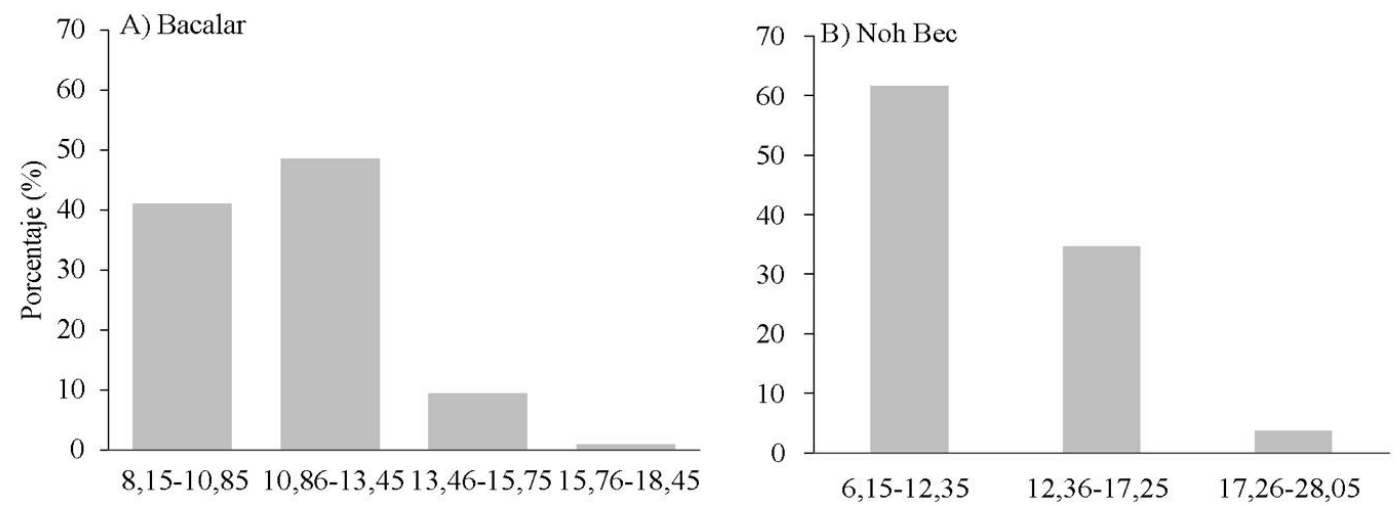

Figura 3. Estratificación vertical de los árboles (m) en los ejidos Bacalar (A) y Noh Bec (B) con base en el método de partición y asignación de datos generada a partir de medioides. from medioids.

Vertical stratification of trees (m) in the Bacalar (A) and Noh Bec (B) ejidos based on the method of data partition and allocation generated
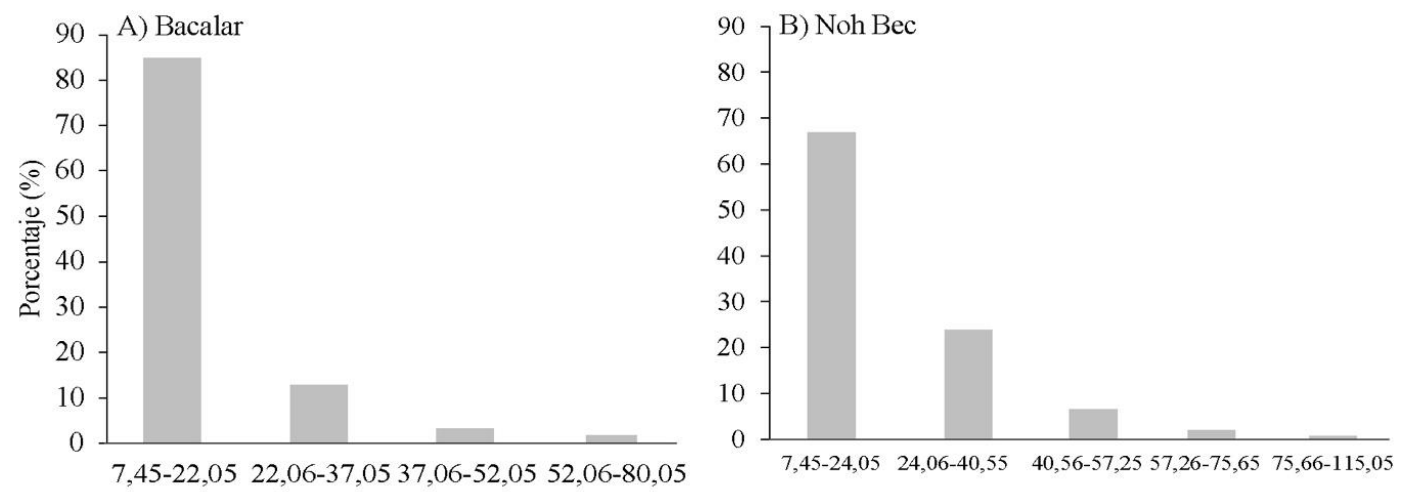

Figura 4. Categorización diamétrica $(\mathrm{cm})$ en los ejidos Bacalar $(\mathrm{A})$ y Noh Bec $(\mathrm{B})$ con base en el método de partición y asignación de datos generada a partir de medioides.

Diametric categorization of the trees $(\mathrm{cm})$ in the Bacalar $(\mathrm{A})$ and Noh Bec (B) ejidos based on the method of data partition and allocation generated from medioids. 
Los valores en DN el área forestal permanente Noh Bec variaron de $7,5 \mathrm{~cm}$ hasta $115 \mathrm{~cm}$, con un diámetro promedio de $21,4 \mathrm{~cm}$ y 625 individuos por hectárea. En este ejido se registraron cinco categorías diamétricas: dos delgadas $(7,45-40,55)$ y tres gruesas $(40,56-115,05)$ (coeficiente de silueta de 0,54) (figura 4B). En las categorías delgadas se acumula el $90,8 \%$ de la densidad.
Importancia estructural. Se aprecia que la acumulación de los valores del IVI e IVMF para las 30 especies más importantes fue parecida entre Noh Bec (IVI $=76,7 \%$ e IVMF $=84,8 \%)$ y Bacalar $(\mathrm{IVI}=84,8 \%$ e IVMF $=87,9 \%)$ (cuadro 1). No obstante, las especies no fueron las mismas en los tres primeros lugares al considerar ambos índices. Las cinco especies con mayor importancia estructural en

Cuadro 1. Índices de valor de importancia (IVI) y valor para el manejo forestal (IVMF) de las especies de las áreas forestales permanentes de los ejidos Bacalar (B) y $\operatorname{Noh} \operatorname{Bec}(\mathrm{N})$.

Importance value and Forest management value indices of the species of the Permanent Forest Areas of the Bacalar and Noh Bec ejidos.

\begin{tabular}{|c|c|c|c|c|c|}
\hline \multirow{2}{*}{ Especie } & \multirow{2}{*}{ Familia } & \multicolumn{2}{|c|}{ IVI } & \multicolumn{2}{|c|}{ IVMF } \\
\hline & & $\mathrm{B}$ & $\mathrm{N}$ & $\mathrm{B}$ & $\mathrm{N}$ \\
\hline Bursera simaruba (L.) Sarg. & Burseraceae & $16,6^{*}$ & 3,4 & $18,1^{*}$ & 3,6 \\
\hline Vitex gaumeri Greenm. & Lamiaceae & $7,7^{*}$ & 2,3 & $7,1^{*}$ & 2,5 \\
\hline Lysiloma latisiliquum (L.) Benth. & Fabaceae & $7,2 *$ & 0,9 & $9,9 *$ & 1,0 \\
\hline Metopium brownei (Jacq.) Urb. & Anacardiaceae & 5,6 & 3,1 & 5,4 & 3,1 \\
\hline Lonchocarpus castilloi Standl. & Fabaceae & 4,2 & 0,1 & 4,8 & 0,1 \\
\hline Manilkara zapota (L.) P. Royen & Sapotaceae & 3,7 & $11,4^{*}$ & 3,9 & $18,1^{*}$ \\
\hline Coccoloba spicata Lundell & Polygonaceae & 3,2 & 0,9 & 2,8 & 0,7 \\
\hline Croton reflexifolius Kunth & Euphorbiaceae & 3,2 & 0,3 & 2,5 & 0,1 \\
\hline Spondias mombin L. & Anacardiaceae & 3,1 & 0,5 & 3,9 & 0,3 \\
\hline Piscidia piscipula (L.) Sarg. & Fabaceae & 2,8 & 1,3 & 2,9 & 1,3 \\
\hline Dendropanax arboreus (L.) Decne. et Planch. & Araliaceae & 2,1 & 3,2 & 2,4 & 3,2 \\
\hline Simarouba amara DC. & Simaroubaceae & 2,0 & 2,7 & 1,8 & 2,4 \\
\hline Hippocratea celastroides H.B.K. & Celastraceae & 2,0 & - & 1,7 & - \\
\hline Brosimum alicastrum $\mathrm{Sw}$. & Moraceae & 1,9 & $6,6^{*}$ & 1,8 & $8,6^{*}$ \\
\hline Pouteria campechiana (Kunth) Baehni & Sapotaceae & 1,8 & 3,8 & 1,6 & 3,7 \\
\hline Protium copal (Schltdl. et Cham.) Engl. & Burseraceae & 1,7 & 2,2 & 1,6 & 1,4 \\
\hline Pouteria reticulata (Engl.) Eyma & Sapotaceae & 1,5 & $13,5^{*}$ & 1,2 & $14,8^{*}$ \\
\hline Exothea diphylla (Standl.) Lundell & Sapindaceae & 1,5 & 0,2 & 1,4 & 0,1 \\
\hline Chrysophyllum mexicanum Brandegee ex Standl. & Sapotaceae & 1,4 & 0,6 & 1,1 & 0,3 \\
\hline Guettarda combsii Urb. & Rubiaceae & 1,2 & 2,3 & 0,9 & 2,0 \\
\hline Nectandra salicifolia (Kunth) Nees & Lauraceae & 1,1 & 0,8 & 0,9 & 0,5 \\
\hline Swartzia cubensis (Britton \& Wilson) Standl. & Fabaceae & 1,1 & 1,5 & 1,7 & 1,7 \\
\hline Simira salvadorensis (Standl.) Standl. & Rubiaceae & 1,1 & 4,2 & 0,9 & 4,3 \\
\hline Eugenia axillaris (Sw.) Willd. & Myrtaceae & 1,0 & 0,0 & 1,0 & 0,0 \\
\hline Alseis yucatanensis Standl. & Rubiaceae & 1,0 & 4,8 & 0,8 & 4,6 \\
\hline Zuelania guidonia (Sw.) Britton et Millsp. & Salicaceae & 1,0 & 0,3 & 0,9 & 0,2 \\
\hline Pseudobombax ellipticum (Kunth) Dugand & Malvaceae & 1,0 & 2,6 & 1,2 & 3,2 \\
\hline Haematoxylum campechianum $\mathrm{L}$. & Fabaceae & 0,9 & - & 0,8 & - \\
\hline Luehea speciosa Willd. & Malvaceae & 0,9 & 1,0 & 0,9 & 0,7 \\
\hline Lonchocarpus xuul Lundell & Fabaceae & 0,5 & 0,0 & 0,9 & 0,0 \\
\hline Swietenia macrophylla King & Meliaceae & 0,8 & 2,0 & 1,1 & 2,4 \\
\hline Subtotal especies (30 Bacalar y 28 Noh Bec) & & 84,8 & 76,7 & 87,9 & 84,8 \\
\hline Especies restantes (56 Bacalar y 78 Noh Bec) & & 15,2 & 23,3 & 12,1 & 15,2 \\
\hline Total de especies (86 Bacalar y 106 Noh Bec) & & 100,0 & 100,0 & 100,0 & 100,0 \\
\hline
\end{tabular}

-: especies ausentes en el ejido N. *: las tres especies con los mayores valores por índice y ejido. IVI: índice de valor de importancia (IVI); IVMF: índice de valor para el manejo forestal (IVMF).

-: Species not registered in ejido N. Values in bold indicate the three species with the highest values by index and ejido. IVI: Importance value index. IVMF: Forest management value index. 
Bacalar fueron: Bursera simaruba (Burseraceae), Vitex gaumeri (Lamiaceae), Lysiloma latisiliquum (Fabaceae), Metopium brownei (Anacardiaceae) y Lonchocarpus castilloi (Fabaceae). En Noh Bec las especies más importantes fueron: Pouteria reticulata (Sapotaceae), Manilkara zapota (Sapotaceae), Brosimum alicastrum (Moraceae), Alseis yucatanensis (Rubiaceae) y Simira salvadorensis (Rubiaceae) (cuadro 1).

Diversidad de especies. La riqueza y repartición de especies fue mayor en el área forestal permanente Noh Bec $\left(\mathrm{D}_{\alpha}=12,07 ; \mathrm{E}=0,78\right)$ en comparación con el área forestal permanente Bacalar $\left(D_{\alpha}=9,61 ; E=0,72\right)$. Los valores del índice de Shannon-Wiener fueron de 3,48 en Bacalar y de 3,37 en Noh Bec, pero no se encontraron diferencias significativas $(P>0,05)$ en la diversidad arbórea entre ejidos.

El valor de alfa de Fisher en Bacalar fue de 15,05, con un límite inferior de 13,71 y un superior igual a 16,38, el error estándar fue de 0,46 y la varianza 0,68 . En tanto, para Noh Bec este índice alcanzó 14,79 , con un límite inferior de 13,77 y un superior igual a 15,8 , con un error estándar de 0,26 y una varianza de 0,51 ; lo que comprueba que la diversidad alfa de Fisher entre ejidos es igual. El índice de Sorensen sugiere un $60,4 \%$ de semejanza entre estas dos áreas forestales permanentes. En el ejido Bacalar, 28 de las especies no se encuentran presentes en Noh Bec, en tanto que en el ejido Noh Bec 48 de las especies no se observan en Bacalar. De manera que 58 especies de las 134 que se registraron en el estudio se comparten entre ejidos.

\section{DISCUSIÓN}

Riqueza y composición en Bacalar y Noh Bec. La riqueza observada entre las áreas forestales permanentes de los ejidos de Bacalar (86 especies) y Noh Bec (106 especies) es diferente, contrario a la hipótesis planteada. Un hallazgo relevante es que aun cuando existen diferencias en la riqueza entre ejidos, el valor de este parámetro para Bacalar y Noh Bec es igual o mayor a lo encontrado en algunas selvas subcaducifolias y caducifolias no intervenidas de la Península de Yucatán y áreas adyacentes (Navarro et al. 2012, Carreón y Valdez 2014, Dzib et al. 2014); pero menor a lo consignado para otras selvas de México y Centroamérica con aprovechamiento maderable (Finegan y Camacho 1999, Gutiérrez-Granados et al. 2011) y sin él (Bongers et al. 1988, Zamora et al. 2008, GutiérrezBáez et al. 2011). Esto sugiere que las selvas estudiadas de Quintana Roo albergan una riqueza moderada, aun cuando se ha sugerido que esta entidad posee el mayor número de taxa arbóreos y endemismos de la Península de Yucatán (Ibarra et al. 1995).

Las familias (Fabaceae, Sapotaceae, Rubiaceae y Sapindaceae) y géneros (Lonchocarpus, Pouteria, Coccoloba, Psidium, Byrsonima, Eugenia, Caesalpinia) mejor representados por número de especies en este estudio coinciden con aquellos característicos de las selvas altas y medianas subcaducifolias y perennifolias poco intervenidas de México (Pennington y Sarukhán 2005, Vester y Navarro 2007), e incluso con los registrados en selvas bajo manejo forestal (Macario et al. 1995, Gutiérrez-Granados et al. 2011). Esto puede deberse a la continua perturbación por incendios, huracanes y aprovechamiento selectivo en estas selvas (Whigham et al. 2003, Navarro et al. 2012), que ha contribuido a una alta densidad de algunos grupos de pioneras con importancia ecológica y comercial adaptadas a las condiciones ambientales presentes en el interior y en los bordes de los claros, como las leguminosas y sapotáceas (Koyoc et al. 2015).

La variación en el comportamiento del número de especies y la estabilidad del ensamblaje arbóreo entre selvas tropicales con y sin aprovechamiento puede ser explicada también debido a que la riqueza es un parámetro dependiente de la escala de medición y del esfuerzo de muestreo (Imai et al. 2012), mientras que la composición es una métrica afectada en principio por la intensidad y el tipo de disturbio (Guariguata y Ostertag 2001).

Aprovechamientos forestales y efectos en la estructura de la vegetación. En relación a los parámetros estructurales, el área basal registrada en el área forestal permanente del ejido de Noh Bec $\left(23,9 \mathrm{~m}^{2} \mathrm{ha}^{-1}\right)$ supera al valor de Bacalar $\left(7,3 \mathrm{~m}^{2} \mathrm{ha}^{-1}\right)$ en este estudio y al de otras selvas aprovechadas de Quintana Roo (1,6 - 7,2 $\mathrm{m}^{2} \mathrm{ha}^{-1}$; Gutiérrez-Granados et al. 2011); pero se ubica en la media con respecto a lo sugerido para árboles con diámetro superior a $3 \mathrm{~cm}$ en selvas intervenidas de Centroamérica $\left(18,6-25,1 \mathrm{~m}^{2} \mathrm{ha}^{-1}\right.$, Finegan y Camacho 1999), Sudamérica (13 - $15 \mathrm{~m}^{2} \mathrm{ha}^{-1}$, Villela et al. 2006) y Asia (19-23 $\mathrm{m}^{2} \mathrm{ha}^{-1}$, Imai et al. 2012).

A su vez, la densidad (284 - 625 individuos ha-1) y el volumen $\left(86,4-183,8 \mathrm{~m}^{3} \mathrm{ha}^{-1}\right)$ presentan resultados superiores a los reportados por Adekunle et al. (2010) para árboles con diámetro mayor a $10 \mathrm{~cm}(132,5$ individuos $\mathrm{ha}^{-1}$ y $\left.14,81 \mathrm{~m}^{3} \mathrm{ha}^{-1}\right)$. Este comportamiento advierte que las selvas estudiadas en Quintana Roo se encuentran estructuralmente bien desarrolladas, aun cuando presentan aprovechamiento maderable. No obstante, esto puede deberse a que el volumen promedio anual de madera obtenido por aprovechamiento selectivo en la Península de Yucatán es relativamente bajo $\left(1 \mathrm{~m}^{3} \mathrm{ha}^{-1}\right.$; Navarro et al. 2012) comparado con otras selvas neotropicales manejadas $\left(10,1 \mathrm{~m}^{3}\right.$ $\mathrm{ha}^{-1}$; Finegan y Camacho 1999), por lo que estudios experimentales que generen información sobre el impacto del manejo forestal bajo diferentes intensidades de aprovechamiento son necesarios para precisar este tópico.

Villela et al. (2006) indican que el aprovechamiento selectivo reduce el número de árboles de gran porte, y que su efecto tiene una relación directa con la cantidad de biomasa extraída y con la vegetación circundante afectada durante las operaciones silvícolas (Adekunle et al. 2010); aspectos que podrían influir sobre la diferencia en los parámetros dasométricos encontrados para ambos ejidos. Sin embargo, Bacalar tiene autorizado un menor volumen madera- 
ble aprovechable comparado con Noh Bec $\left(10,8 \mathrm{~m}^{3} \mathrm{ha}^{-1}\right.$ vs $23,9 \mathrm{~m}^{3} \mathrm{ha}^{-1}$ de volumen total, respectivamente). Esto indica que, para este caso en particular, el aprovechamiento forestal no es el mejor predictor de la respuesta en los cambios sobre la estructura de la vegetación arbórea en las áreas forestales permanentes, por lo que la calidad de sitio producto de la histéresis que cada ejido ha presentado en el tiempo podría explicar en forma más precisa este comportamiento.

En cambio, a nivel de sotobosque, al igual que la propuesta de Gutiérrez-Granados et al. (2011), se observó que podría existir una relación positiva entre la intensidad del disturbio generado por el aprovechamiento maderable y el porcentaje de especies pioneras registradas en un sitio. Macario et al. (1995) mencionan que existen algunas especies de las selvas manejadas de Quintana Roo que se benefician por la perturbación, tales como Bursera simaruba, Swietenia macrophylla, Pseudobombax ellipticum, Dendropanax arboreus y Metopium brownei; mientras que Simarouba amara presenta incrementos muy reducidos tanto en densidad como en altura. Todas estas especies también son dominantes en las áreas forestales permanentes de los ejidos de Bacalar y Noh Bec, lo que sugiere que la dinámica ecológica posterior a la perturbación producida por el aprovechamiento forestal facilita el establecimiento y crecimiento de estas especies en selvas intervenidas, favoreciendo sus atributos dasométricos.

La estratificación vertical de los árboles en estos ejidos (6-30 m, promedio $12 \mathrm{~m}$ ) se considera que es consistente con el comportamiento fisonómico-florístico consignado por otros autores (Gutiérrez-Báez et al. 2011, Carreón y Valdez 2014, Dzib et al. 2014, Koyoc et al. 2015) para las selvas de la región, el cual está representado por dos a tres estratos con $\sim 90 \%$ del total de individuos agrupados en categorías menores a $12 \mathrm{~m}$. De hecho, Pennington y Sarukhán (2005) indican que la altura de las selvas medianas subcaducifolias del país oscila entre 15 y $30 \mathrm{~m}$ y distinguen tres estratos conformados por diferentes especies: superior $>25 \mathrm{~m}$, medio 13-24 $\mathrm{m}$ e inferior 5-12 m.

Los mismos autores refieren que Vitex gaumeri, Brosimum alicastrum, Swietenia macrophylla, Pseudobombax ellipticum, Lysiloma latisiliquum y Manilkara zapota dominan el dosel superior de estas selvas; mientras que Alseis yucatanensis y Spondias mombin están presentes en el dosel intermedio y en el sotobosque (Pennington y Sarukhán 2005). A pesar de estas tendencias, es evidente que el aprovechamiento forestal tiene un efecto diferencial en la distribución de los individuos y en la formación de estratos en ambas selvas ejidales; lo que sugiere que la selva de Bacalar ha sido objeto de mayor perturbación, lo cual ha favorecido la conformación de un elevado número de estratos y grupos funcionales adaptados a las condiciones restrictivas de cada uno de estos. Se sabe que en las selvas la luz es un factor limitativo en el desarrollo de las especies arbóreas y la estratificación es la solución a esta limitación (Guariguata y Ostertag 2001).
La estructura diamétrica de las selvas en los ejidos estudiados sigue un patrón contrario al de la distribución vertical, ya que en general Noh Bec tiene una mejor representación en diámetro del arbolado, al alcanzar algunos de los árboles registrados más de $115 \mathrm{~cm}$; lo cual es consistente con el área basal registrada para este ejido. Pese a ello, alrededor del $80 \%$ de los individuos en ambas selvas tienen diámetros menores a $22,5 \mathrm{~cm}$, consecuente con el patrón tipo I de distribución horizontal propuesto por Bongers et al. (1988), en donde existe un número elevado de individuos en las clases diamétricas más pequeñas, que tiende a disminuir con rapidez en las clases intermedias y se reduce a niveles muy bajos en las clases diamétricas mayores $(\geq 40 \mathrm{~cm})$.

En consecuencia, la tendencia de las frecuencias diamétricas del arbolado en las selvas de Bacalar y Noh Bec asemeja a una curva en forma de $\mathrm{J}$ invertida, característica de selvas incoetáneas, que se expresa en forma de exponencial negativa, es decir descendente de menor a mayor diámetro (Gutiérrez-Báez et al. 2011). Este comportamiento se ha mencionado en otras selvas aprovechadas (Adekunle et al. 2010, Koyoc et al. 2015), lo que sugiere que el aprovechamiento forestal selectivo favorece la existencia de condiciones ecológicas que estimulan una buena reproducción, establecimiento y repoblación natural continua de las especies arbóreas, sobre todo al dejar árboles semilleros en las áreas bajo manejo (Günter et al. 2011).

Es importante mencionar que aun cuando los resultados estructurales del presente estudio son semejantes a los encontrados por otros autores (Pennington y Sarukhán 2005, Gutiérrez-Báez et al. 2011, Carreón y Valdez 2014, Dzib et al. 2014, Koyoc et al. 2015), el método utilizado en todos estos casos no emplea criterios cuantitativos para la estratificación vertical, lo que resulta en clasificaciones arbitrarias de la altura del arbolado para la diferenciación de estratos, en particular en cuanto al número y amplitud de los intervalos de clase. Debido a tales inconsistencias, este trabajo propuso un nuevo método basado en la partición por medioides (Kaufman y Rousseeuw 2005) pero aplicado a la estimación de estadísticas de los puntos de inflexión que marcan diferencias entre estratos. Debido a la facilidad de los datos requeridos para su construcción y al diseño analítico robusto, se sugiere que este método pueda aplicarse tanto en selvas con aprovechamiento maderable como en aquellas bajo conservación, a fin de aportar información que sustente el manejo y cuidado de las selvas del Neotrópico.

Con respecto a la importancia estructural de las especies, la dominancia de algunos taxa arbóreos advierte diferencias notorias tanto a nivel de ejidos como por el índice utilizado, lo que explica la presencia de una composición arbórea particular entre ambas selvas, aun cuando la semejanza florística es del orden de $60 \%$. La especie dominante en Noh Bec fue Pouteria reticulata (IVI = 13,5), semejante a lo encontrado por Carreón y Valdez (2014) en selvas serales unos $20 \mathrm{~km}$ al este del área de estudio. En cambio, 
Bursera simaruba $(\mathrm{IVI}=16,6)$ fue la especie mejor representada en Bacalar, similar a lo registrado por Zamora et al. (2008) y Gutiérrez-Báez et al. (2011) para las selvas medianas subcaducifolias de Yucatán. De hecho, las tres especies más importantes de acuerdo con el IVI en los dos ejidos son taxones dominantes de las selvas medianas subcaducifolias del sureste de México (Zamora et al. 2008, Navarro et al. 2012, Dzib et al. 2014).

Un hallazgo importante de este estudio es que $24 \mathrm{de}$ las 30 especies arbóreas registradas con mayor IVI en Bacalar y Noh Bec son también un componente característico en selvas poco alteradas de la Península de Yucatán (Gutiérrez-Báez et al. 2011, Dzib et al. 2014); nueve de las cuales son utilizadas, con regularidad, para aprovechamientos maderables (Vester y Navarro 2007). En tanto que Bursera simaruba, Metopium brownei, Coccoloba spicata, Dendropanax arboreus, Pseudobombax ellipticum y Swietenia macrophylla son especies serales tempranas de vida larga, típicas de selvas perturbadas (Gutiérrez-Granados et al. 2011, Carreón y Valdez 2014, Dzib et al. 2014). Lo anterior enfatiza el grado de conservación de estas selvas ejidales con manejo forestal, debido al predominio de especies representativas de etapas serales avanzadas, contrario a lo indicado por Gutiérrez-Granados et al. (2011) en las selvas manejadas en Felipe Carrillo Puerto, Quintana Roo.

El índice de valor para el manejo forestal (IVMF) en el área forestal permanente del ejido Bacalar mostró un ajuste en las posiciones de las especies que ocupaban los sitios 2 y 3 de acuerdo con IVI (Vitex gaumeri y Lysiloma latisiliquum), sin afectar el predominio de Bursera simaruba. En cambio, en Noh Bec la reordenación de especies fue más evidente, dado que la especie mejor representada con el IVI, Pouteria reticulata, fue sustituida por Manilkara zapota. Esto puede deberse a que el IVMF jerarquiza la importancia estructural cuando se agrega el volumen total de árboles en sustitución del valor de la frecuencia relativa. De manera que el IVI es un índice sintético que solo pondera el predominio de cada especie con base en diferentes atributos dasométricos en el plano horizontal (Carreón y Valdez 2014); en tanto el IVMF integra una variable compuesta a esta ecuación, volumen total árbol, obtenida a partir de parámetros estructurales en sentido vertical y horizontal.

Por lo tanto, se considera que el IVMF tiene la ventaja de servir como una herramienta para la evaluación forestal continua en áreas bajo aprovechamiento forestal maderable, ya que permite definir tanto la estructura óptima para intensificar las cortas en especies no deseables para el manejo forestal, como conservar las especies en estado crítico en función de su bajo predominio. Cabe señalar que, de las 10 especies jerarquizadas mediante el IVMF en Bacalar, solo Spondias mombin, Coccoloba spicata y Croton reflexifolius carecen de importancia en el mercado nacional, mientras que en Noh Bec todas las especies son de interés maderable (Vester y Navarro 2007).
Diversidad arbórea en selvas manejadas. De acuerdo con la hipótesis planteada, los valores de diversidad arbórea registrados en Bacalar $\left(\mathrm{H}^{\prime}=3,48 ; \alpha=17,09\right)$ y Noh Bec $\left(H^{\prime}=3,37 ; \alpha=15,26\right)$ son estadísticamente iguales, e incluso son similares o mayores a los referidos para selvas tropicales sin intervención en Quintana Roo $\left(\mathrm{H}^{\prime}=2,78-\right.$ 3,33; Carreón y Valdez 2014), Yucatán ( $H^{\prime}=3,55-4,82$; Zamora et al. 2008) y Campeche ( $\mathrm{H}^{\prime}=1,62-2,77$; Dzib et al. 2014). De hecho, los resultados del presente estudio se asemejan a los de otras selvas bajo manejo forestal en África ( $\mathrm{H}^{\prime}=3,03$; Adekunle et al. 2010) y Latinoamérica $\left(\mathrm{H}^{\prime}=2,10-2,60\right.$, Gutiérrez-Granados et al. 2011). Esto confirma que el aprovechamiento maderable es una alternativa factible de manejo en estas selvas, debido a que no tiene un efecto sobre la diversidad, aun cuando puede influir en la estructura y composición de especies. Planteamientos semejantes han sido hechos por Villela et al. (2006) en selvas estacionalmente secas de Brasil, donde no encontraron efecto del aprovechamiento selectivo en la diversidad de especies.

Finalmente, es importante resaltar que a pesar de que ambas selvas ejidales tienen una historia de aprovechamiento forestal semejante y presentan el mismo tipo de vegetación, difieren en cuanto a valores de riqueza y estructura. Por lo que se plantea que una mayor incidencia de huracanes en Bacalar podría haber determinado estas diferencias en los atributos estructurales entre ejidos (Navarro y Granados 1997), ya que a decir de Whigham et al. (2003), los huracanes afectan la dinámica natural de las selvas debido a que ocasionan incrementos en las tasas de mortalidad, incorporación y crecimiento de las poblaciones y, en consecuencia, modifican diversos atributos ecológicos.

Se ha documentado que en las selvas intervenidas de Quintana Roo afectadas por el huracán Dean, se redujo la altura de los árboles residuales en un $16 \%$ y el área basal en 4,5\% (Navarro et al. 2012). En tanto que, en sitios permanentes de investigación silvícola localizados en el ejido de Bacalar, se demostró que la intensidad del viento producida por el huracán Gilberto afectó en forma negativa la composición florística, el área basal, la densidad y la diversidad de especies (Navarro y Granados 1997).

\section{CONCLUSIONES}

Las selvas de Bacalar y Noh Bec muestran diferencias en la estructura, composición y riqueza de especies, no así en la diversidad, aun cuando comparten el mismo tipo de vegetación y un historial semejante de intervención forestal. Por lo tanto, se considera que las prácticas silvícolas asociadas al historial de disturbios naturales son algunos de los factores que determinan la variación en estos atributos ecológicos en las selvas estudiadas. A su vez, se propone el IVMF como un estimador robusto y de amplia utilidad para estudios de ecología y silvicultura tropical, ya que proporciona información cuantitativa sobre la importancia silvícola de las especies arbóreas con condiciones 
deseables para el manejo forestal. De igual forma, se considera que el método de partición de medioides aplicado al ámbito forestal permite una aproximación más certera para la determinación de los puntos de inflexión y de la definición del número de estratificaciones diamétricas y de alturas que forman las masas forestales, información que tiene implicaciones directas en las operaciones silvícolas y que permitirá profundizar en el conocimiento para un mejor uso y conservación de las selvas tropicales del Neotrópico.

\section{AGRADECIMIENTOS}

A Trópica Rural Latinoamericana, A.C. por el apoyo en el registro de datos en campo. Al personal de campo de los ejidos Noh Bec y Bacalar.

\section{REFERENCIAS}

Adekunle V, A Olagoke, L Ogundare. 2010. Logging Impacts in Tropical Lowland Humid Forest on Tree Species Diversity and Environmental Conservation. Journal of Sustainable Forestry 29(5): 517-538. DOI: https://doi.org/10.1080/105 $\underline{49811.2010 .489923}$

Bongers F, J Popma, J Meave del Castillo, J Carabias. 1988. Structure and floristic composition of the lowland rain forest of Los Tuxtlas, Mexico. Vegetatio 74(1): 55-80. DOI: https://doi.org/10.1007/BF00045614

Bray D. 2004. Community forestry as a strategy for sustainable management: Perspectives from Quintana Roo, Mexico. In Zarin D, J Alavalapati, F Putz, M Schmink eds. Working forests in the American tropics. New York, USA. Columbia University Press. p. 221-237.

Carreón R, J Valdez. 2014. Estructura y diversidad arbórea de vegetación secundaria derivada de una selva mediana subperennifolia en Quintana Roo. Revista Chapingo Serie Ciencias Forestales y del Ambiente 20(1): 119-130. DOI: http://dx.doi.org/10.5154/r.rchscfa.2013.06.023.

Chazdon R. 2013. Tropical forest regeneration. In Levin S eds. Encyclopedia of Biodiversity. Waltham, USA. Academic Press. p. 277-286.

CONAFOR (Comisión Nacional Forestal, MX). 2012. Inventario Nacional Forestal y de Suelo. Informe 2004-2009. Comisión Nacional Forestal y Secretaría de Medio Ambiente y Recursos Naturales. Consultado 19 mar. 2019. Disponible en https:/www.cnf.gob.mx:8443/snif/portal/infys/temas/ resultados-2004-2009

Dzib B, C Chanatásig, N González. 2014. Estructura y composición en dos comunidades arbóreas de la selva baja caducifolia y mediana subcaducifolia en Campeche, México. Revista Mexicana de Biodiversidad 85(1): 167-178. DOI: http://dx.doi.org/10.22201/ib.20078706e.2014.1.88

Finegan B, M Camacho. 1999. Stands dynamics in a logged and silviculturally treated Costa Rican rain forest, 1988-1996. Forest Ecology and Management 121(3): 177-189. DOI: https://doi.org/10.1016/S0378-1127(98)00550-7

Gadow V, S Sánchez, J Álvarez. 2007. Estructura y crecimiento del bosque. Göttingen, Alemania Universidad de Göetingen. $147 \mathrm{p}$.

Guariguata R, R Ostertag. 2001. Neotropical secondary forest succession: changes in structural and functional characteristics. Forest Ecology and Management 148(1): 185-206.

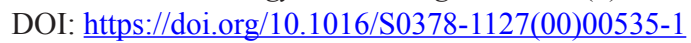

Günter S, M Weber, B Stimm, R Mosandl. 2011. Silviculture in the Tropics. Freising, Germany. Springer-Verlag Berlin Heidelberg. 559 p.

Gutiérrez-Báez C, J Ortiz-Díaz, J Flores, P Zamora, M Domínguez, P Villegas. 2011. Estructura y composición florística de la selva mediana subcaducifolia de Nohalal-Sudzal Chico, Tekax, Yucatán, México. Foresta Veracruzana 13(1): 7-14.

Gutiérrez-Granados G, D Pérez-Salicrup, R Dirzo. 2011. Differential diameter-size effects of forest management on tree species richness and community structure: implications for conservation. Biodiversity and Conservation 20(7): 15711585. DOI: https://doi.org/10.1007/s10531-011-0046-8

Ibarra G, J Villaseñor, R Duran. 1995. Riqueza de especies y endemismo del componente arbóreo de la Península de Yucatán, México. Boletín de la Sociedad Botánica de México 57: 49-77. DOI: http://dx.doi.org/10.17129/botsci.1476

Imai N, T Seino, S Aiba, M Takyu, J Titin, K Kitayama. 2012. Effects of selective logging on tree species diversity and composition of Bornean tropical rain forests at different spatial scales. Plant Ecology 213(9): 1413-1424. DOI: https://doi.org/10.1007/s11258-012-0100-y

INEGI (Instituto Nacional de Estadística y Geografía, MX). 2017. Anuario Estadístico y Geográfico de Quintana Roo 2017. INEGI, México. Consultado 20 ene. 2018. Disponible en http://internet.contenidos.inegi.org.mx/contenidos/ Productos/prod_serv/contenidos/espanol/bvinegi/productos/nueva estruc/anuarios 2017/702825095130.pdf

Kaufman L, P Rousseeuw. 2005. Finding groups in data: an introduction to cluster analysis. New York, USA. John Wiley. $342 \mathrm{p}$.

Koleff P, T Urquiza, B Contreras. 2012. Prioridades de conservación de los bosques tropicales en México: reflexiones sobre su estado de conservación y manejo. Ecosistemas 21(1-2): 6-20.

Koyoc L, J Mendoza, J Pérez, N Torrescano. 2015. Efectos de perturbación antrópica en Petenes de selva en Campeche, México. Acta Botánica Mexicana (110): 89-103. DOI: https://doi.org/10.21829/abm110.2015.189

Macario P, E García, J Aguirre, E Hernández. 1995. Regeneración natural de especies arbóreas en una selva mediana subperennifolia perturbada por extracción forestal. Acta Botánica Mexicana 32: 11-23. DOI: https://doi.org/10.21829/ abm32.1995.742

Magurran A, B McGill. 2014. Biological Diversity: Frontiers in Measurement and Assessment. New York, USA. Oxford University Press. 359 p.

Navarro M, D Granados. 1997. Dinámica sucesional en un bosque tropical afectado por disturbios en la zona norte de Quintana Roo. Revista Chapingo Serie Ciencias Forestales 1: 5-16.

Navarro M, R Durán, M Méndez. 2012. El impacto del huracán Dean sobre la estructura y composición arbórea de un bosque manejado en Quintana Roo, México. Madera y Bosques 18(1): 57-76. DOI: https://doi.org/10.21829/ myb.2012.1811138

Pennington T, J Sarukhán. 2005. Árboles tropicales de México. Distrito Federal, México. Fondo de Cultura Económica. $523 \mathrm{p}$.

Velasco E, M Ramírez, S Moreno, V De la Rosa. 2003. Estima- 
dores de razón para el inventario nacional forestal de México. Revista Ciencia Forestal en México 28(94): 23-44.

Vester H, M Navarro. 2007. Árboles maderables de Quintana Roo. Distrito Federal, México. ECOSUR, COQCYT, Gobierno del Estado de Quintana Roo, CONABIO. 139 p.

Villela D, M Nascimiento, L De Arago, D Da Gama. 2006. Effect of selective logging on forest structure and nutrient cycling in a seasonally dry Brazilian Atlantic forest. Journal of Biogeography 33(3): 506-516. DOI: https://doi.org/10.1111/ j.1365-2699.2005.01453.x

Whigham D, I Olmsread, E Cabrera, A Curtis. 2003. Impacts of hurricanes of the forests of Quintana Roo, Yucatan Peninsula, México. In Gómez A, M Allen, S Fedick, J Jiménez eds. Lowland Maya Area: Three Millenia at the human-wildland interface. New York, USA. Haworth Press. p. 193-212.

Zamora P, G García, J Flores, J Ortiz. 2008. Estructura y composición florística de la selva mediana subcaducifolia en el sur del estado de Yucatán, México. Polibotánica (26): 39-66.

Recibido: $19 / 10 / 18$

Aceptado: 26/03/19 